\title{
Índice de falha diagnóstica na detecção de fraturas e luxações perilunares do carpo utilizando radiografias simples do punho*
}

\section{Diagnostic Failure Rate in Detecting Perilunate Carpal Fractures and Dislocations Using Plain Wrist X-Rays}

\footnotetext{
1 Programa de Cirurgia da Mão, Hospital das Clínicas, Faculdade de Medicina de Ribeirão Preto, Universidade de São Paulo (HC-FMRPUSP), Ribeirão Preto, SP, Brasil

2 Divisão de Cirurgia da Mão, Hospital das Clínicas, Faculdade de Medicina de Ribeirão Preto, Universidade de São Paulo (HC-FMRPUSP), Ribeirão Preto, SP, Brasil
}

Aleixo Abreu Tanure ${ }^{1}$ Fernanda Ruiz de Andrade ${ }^{1}$ Luis Guilherme Rosifini Alves Rezende ${ }^{10}$

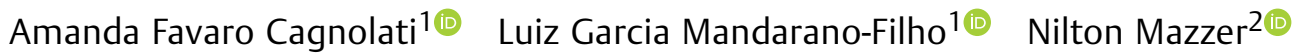

Endereço para correspondência Luis Guilherme Rosifini Alves Rezende, MD, Av. Bandeirantes, 3900, Vila Monte Alegre, Ribeirão Preto, SP, 14049-900, Brasil (e-mail: lugui.rezende@yahoo.com.br).

Rev Bras Ortop 2021;56(3):340-345.

\author{
Resumo \\ Palavras-chave \\ - ossos do carpo/lesões \\ - traumatismos do \\ punho \\ - luxações articulares \\ - fraturas ósseas
}

Objetivos O presente estude teve como objetivo avaliar o índice de falha diagnóstica na detecção de fraturas e luxações perilunares do carpo utilizando radiografias simples do punho por ortopedistas e residentes de ortopedia. Secundariamente, identificar possíveis grupos que apresentem maior ou menor chance de acerto diagnóstico.

Métodos Foi aplicado um questionário online a diversos ortopedistas através de email, redes sociais e aplicativos de comunicação via smartphone, para avaliar o índice de falha diagnóstica na detecção de fraturas e luxações perilunares utilizando radiografias simples.

Resultados Foram obtidas 511 respostas e observado um índice de erro diagnóstico de $8,81 \%$ para as luxações simples e $1,76 \%$ para fratura transescafoperilunar. Ao estratificar por grupos, os médicos residentes obtiveram os maiores índices de erro nas luxações perilunares simples (23,91\%), já os cirurgiões de mão obtiveram os índices mais baixos $(1,74 \%)$.

Conclusão Ao comparar com a literatura, os índices de falha encontrados foram menores, sugerindo que a radiografia simples é eficaz e que o índice de erro pode não ser tão elevado quanto o relatado na literatura.
Trabalho realizado no Hospital das Clínicas da Faculdade de Medicina de Ribeirão Preto da Universidade de São Paulo, Ribeirão Preto, SP, Brasil. recebido

05 de Fevereiro de 2020

aceito

05 de Maio de 2020

Publicado on-line

Setembro 25, 2020
DOI https://doi.org/ $10.1055 / \mathrm{s}-0040-1714227$ ISSN $0102-3616$.
(C) 2020. Sociedade Brasileira de Ortopedia e Traumatologia. All rights reserved.

This is an open access article published by Thieme under the terms of the Creative Commons Attribution-NonDerivative-NonCommercial-License, permitting copying and reproduction so long as the original work is given appropriate credit. Contents may not be used for commercial purposes, or adapted, remixed, transformed or built upon. (https://creativecommons.org/ licenses/by-nc-nd/4.0/)

Thieme Revinter Publicações Ltda., Rua do Matoso 170, Rio de Janeiro, RJ, CEP 20270-135, Brazil 
Abstract
Keywords
- carpal bones/injuries
- wrist injuries
- joint dislocations
- fractures, bone

Objectives The present study aimed to evaluate the diagnostic failure rate in detecting perilunate fractures and dislocations using plain wrist radiographs by orthopedists and orthopedic residents. A secondary objective was to identify possible groups with a greater or lesser chance of establishing a correct diagnosis.

Methods An online questionnaire was sent to several orthopedists through e-mail, social networks, and smartphone-based communication applications to assess the rate of diagnostic failure in detecting perilunate fractures and dislocations using plain radiographs. Results A total of 511 responses was obtained, with a diagnostic error rate of $8.81 \%$ for simple dislocations and $1.76 \%$ for trans-scaphoid perilunate fractures. Group stratification showed that residents presented the highest error rates in simple perilunate dislocations (23.91\%), whereas hand surgeons presented the lowest error rates (1.74\%).

Conclusion Compared with the literature, the failure rates found were lower, suggesting that plain radiography is effective and that the error rate may not be as high as reported.

\section{Introdução}

As fraturas e luxações perilunares do punho são patologias incomuns, correspondendo a aproximadamente $7 \%$ de todas as lesões carpais, ${ }^{1}$ e apresentam repercussões graves para os pacientes acometidos se não diagnosticados e tratados de forma correta. Essas lesões são decorrentes de traumas de alta energia, tais como acidentes automobilísticos, queda de altura, e esportes de contato e frequentemente associam-se a outras lesões traumáticas. Os pacientes apresentam dor difusa no punho, edema, perda do arco de movimento e posição semifletida dos dedos. Pode ocorrer ainda queixa de parestesias no território do nervo mediano e síndrome do túnel do carpo aguda. ${ }^{2}$

A avaliação radiográfica é essencial para o manejo desses pacientes, e as radiografias em incidência posteroanterior $(\mathrm{PA}) \mathrm{e}$ de perfil (P) são suficientes para o diagnóstico. A radiografia em incidência PA com desvio ulnar do punho auxilia na avaliação de fraturas-luxações transescafoperilunares. ${ }^{2} \mathrm{Na}$ radiografia em incidência PA, é importante observar as linhas de Gilula, que são linhas imaginárias desenhadas através dos aspectos proximal e distal da fileira proximal e do aspecto proximal da fileira distal. Essas três linhas devem ser arcos suaves e paralelos entre si, e rupturas nessas linhas sugerem incongruência carpal. ${ }^{3} \mathrm{Na}$ incidência de perfil, observa-se o alinhamento do capitato, do semilunar e do rádio. Esses ossos devem estar alinhados, e qualquer alteração do alinhamento sugere fortemente uma luxação perilunar. A tomografia computadorizada pode ser útil quando existem fraturas complexas associadas, como as do escafoide e do piramidal, e deve ser realizada após a redução da luxação. ${ }^{4}$

Mayfield et al., ${ }^{5}$ em 1980, realizaram um estudo em cadáver e classificaram essa patologia em 4 estágios progressivos. Foi aplicada uma força axial com hiperextensão do punho, associada a desvio ulnar e supinação intercarpal para reproduzir a lesão. No estágio I observaram uma ruptura do ligamento escafosemilunar ou uma fratura do escafoide. No estágio II, foi observada a subluxação do semilunocapitato, podendo ocorrer em alguns casos fratura do capitato. No estágio III, houve lesão do ligamento semilunopiramidal ou fratura do piramidal, ocorrendo uma luxação perilunar dorsal de todo o carpo. No estágio IV, observaram a luxação palmar do semilunar para o túnel do carpo no ponto em que o capitato reduz para a fossa do semilunar. ${ }^{5}$

Devido à pouca frequência e baixa familiaridade de grande parte dos ortopedistas com a anatomia complexa dos ossos do carpo, a luxação perilunar, em muitos casos, não é diagnosticada no primeiro atendimento. ${ }^{1,2}$ Em um estudo multicêntrico com 166 pacientes, Herzberg et al. ${ }^{6}$ demonstraram que em $25 \%$ dos casos o diagnóstico não foi realizado na avaliação inicial, tanto nas luxações simples como nas fraturas-luxações transescafoperilunares. Esse dado é preocupante pois o diagnóstico e tratamento precoce é fundamental para minimizar complicações graves como rigidez, dor crônica e artrose pós-traumática, $2,4,7,8$ e o atraso no tratamento comprovadamente afeta de forma negativa o resultado final. ${ }^{6,9}$

Essa realidade relatada nos estudos internacionais não parece diferente da realidade brasileira. Em nosso serviço especializado na cirurgia da mão, recebemos muitos pacientes com luxações perilunares crônicas que receberam um primeiro atendimento por ortopedistas e não foi realizado $o$ diagnóstico, prejudicando o resultado do tratamento.

O objetivo do presente trabalho foi avaliar o índice de falha diagnóstica na detecção de fraturas e luxações perilunares do carpo utilizando radiografias simples do punho por ortopedistas e residentes de ortopedia. Secundariamente, identificar possíveis grupos que apresentem maior ou menor chance de acerto diagnóstico.

\section{Materiais e métodos}

Foi elaborado e aplicado um questionário online confeccionado utilizando a plataforma Google Forms (Google LLC, Menlo Park, CA, USA) ${ }^{10}$ e encaminhado para ortopedistas e residentes de ortopedia através de e-mail, redes sociais (Workplace from Facebook [Facebook Inc., Menlo Park, CA, USA] da Sociedade Brasileira de Ortopedia e Traumatologia) e aplicativos de comunicação (WhatsApp [Facebook Inc.]) 
via smartphone. Estas plataformas foram escolhidas por oferecerem possibilidade de grande alcance do questionário para ortopedistas e residentes de ortopedia e apresentarem comodidade para respondê-lo. O questionário foi composto de quatro perguntas iniciais para analisar o perfil dos objetos do estudo. Essas perguntas foram em relação à quantidade de anos do término da residência médica, área de atuação, presença do título de especialista emitido pela Sociedade Brasileira de Ortopedia e Traumatologia (SBOT), e se o profissional atua em unidade de urgência/emergência que atende trauma ortopédico do membro superior. Na segunda etapa do questionário, foram mostradas oito radiografias em incidência PA e de perfil do punho, sendo três delas normais e cinco delas patológicas. Dentre estas, uma apresentava luxação perilunar simples e outra uma fratura-luxação perilunar. Havia mais três radiografias, duas com a presença de fratura da extremidade distal do rádio e uma com fratura do escafoide. O profissional, após a análise, deveria responder se a radiografia era normal ou se apresentava presença de fratura e/ou luxação. A ordem das radiografias apresentadas era aleatória. Os critérios de exclusão foram questionários respondidos de forma incompleta ou contraditórios. Para verificar a associação entre as variáveis qualitativas, os dados foram submetidos ao teste do Qui-Quadrado. Além disso, a quantificação desta associação foi mensurada por meio de modelos de regressão logística, ${ }^{11}$ onde se calculou a razão de chances $(\mathrm{RC})$ bruta com seus respectivos intervalos de confiança de $95 \%$.

Este trabalho foi aprovado pelo comitê de ética da instituição sob o número CAAE 84365318.3.0000.5440.

\section{Resultados}

Foram obtidas 511 respostas. Dessas, 194 (38\%) de ortopedistas formados há mais de 10 anos, 225 (44\%) de ortopedistas formados há menos de 10 anos, e 92 (18\%) de médicos residentes de ortopedia. Dos 419 ortopedistas formados, 352 (84\%) possuíam título pela SBOT e 67 (16\%) não possuíam título de especialista reconhecido pela SBOT. Foi observado ainda que dos 419 ortopedistas, 172 (41\%) atuam na área de cirurgia da mão, 90 (21,5\%) atuam como ortopedista gerais e 157 (37,5\%) atuam como especialistas de outras áreas como coluna, joelho, ombro, entre outros. Das 511 respostas, 436 afirmaram que trabalham atualmente em unidade de emergência que recebe traumas do membro superior (85,3\%).

Na - Figura 1, a radiografia apresentava uma luxação perilunar simples estágio III de Mayfield. Foi observado um índice de erro diagnóstico de 8,81\%. Entre os residentes, este erro foi de $23,91 \%$ e entre os ortopedistas formados, o erro foi de 5,49\% (-Figura 2). Os médicos residentes apresentaram uma chance de errar ( $R C$ ) 4,3 vezes maior que os ortopedistas formados há mais de 10 anos e 6,7 vezes maior que os formados há menos de 10 anos. Quando comparados por área de atuação, os cirurgiões de mão obtiveram o menor índice de erro, $1,74 \%$. A chance de um ortopedista geral e um ortopedista especialista errar foi cerca de 5 vezes maior que os cirurgiões de mão (RC 0,211 e 0,197, respectivamente). Comparando os residentes com os cirurgiões de mão, a chance de erro foi cerca de 17,5 vezes maior (RC 0,057$)$. Os valores de $p$ foram menores que 0,001 ( - Tabela $\mathbf{1}$ ).

$\mathrm{Na}$ - Figura 3, a radiografia demonstrava a presença de uma fratura transescafoperilunar estágio III. Observou-se um índice

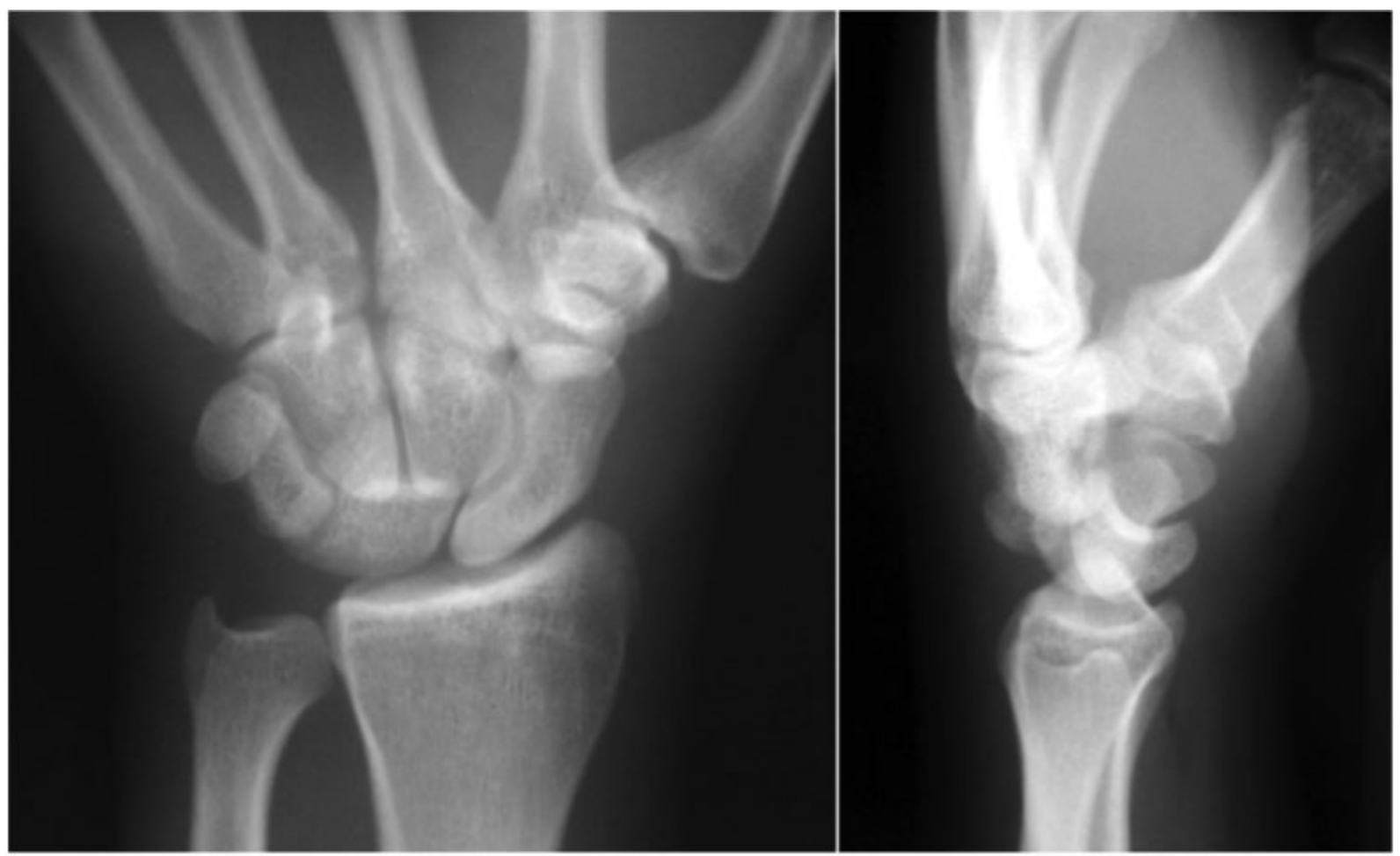

Fig. 1 Luxação perilunar estágio III de Mayfield. 


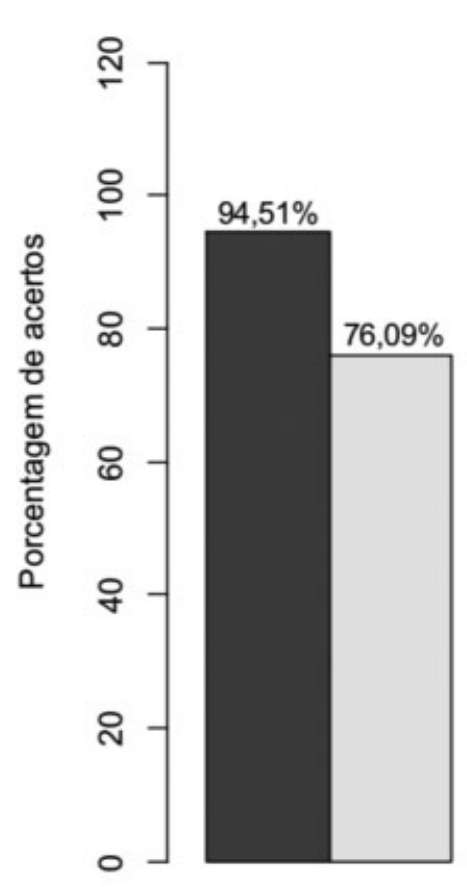

RX1
Não Residente

Residente

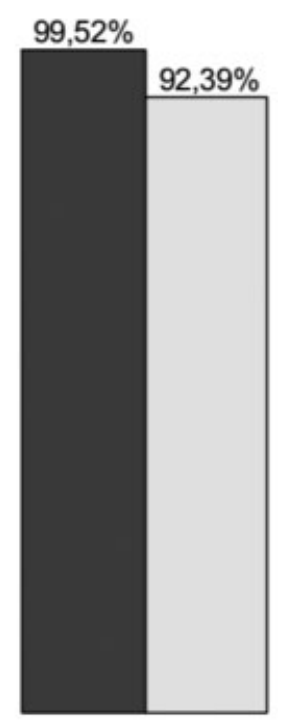

$\mathrm{R} \times 2$

Fig. 2 Porcentagem de acertos: Residente x Não Residente.

de erro diagnóstico global de 1,76\%. Entre os residentes esse erro foi $7,61 \%$, e entre os ortopedistas $0,48 \%$ (-Figura 2 ). Isso representa uma RC de 9,1 para ortopedistas formados a menos de 10 anos e de 3,6 para ortopedistas gerais, com um valor de $p<0,001$. Ortopedistas com mais de 10 anos de formados não apresentaram nenhum erro diagnóstico para essa radiografia, assim como cirurgiões de mão e ortopedistas especialistas (-Tabela 2).

Dentre os ortopedistas formados não houve diferença relevante nas respostas quando separados pela presença de título de especialista pela SBOT. Quanto às radiografias normais apresentadas no questionário, $38,49 \%$ das respostas as classificaram como alteradas.

\section{Discussão}

O estudo apresentou um número de respostas bastante significativo, com boa participação da comunidade ortopédica. As 511 respostas obtidas correspondem a aproximadamente 3\% dos ortopedistas do país. ${ }^{12}$ Entretanto, a amostragem do estudo foi realizada por conveniência, o que pode limitar a validade externa do estudo e não ser necessariamente representativa de todas as regiões geográficas do país.

A maioria dos artigos encontrados na literatura que mencionam o índice de falha diagnóstica da luxação perilunar simples ou complexa citam o estudo de Herzberg et al. ${ }^{6}$ realizado na

Tabela 1 Tabela de frequências e regressão logística para a radiografia 1

\begin{tabular}{|c|c|c|c|c|c|c|}
\hline & \multicolumn{2}{|l|}{ RX1 } & & & & \\
\hline & Erro (0) & Acerto (1) & & & & \\
\hline & $\mathrm{n}(\%)$ & $\mathrm{n}(\%)$ & Total & Valor de $p^{*}$ & Razão de chances bruta & Intervalo de Confiança (95\%) \\
\hline \multicolumn{7}{|c|}{ Pergunta 1: Qual a sua graduação atual? } \\
\hline 1 & $13(2,54)$ & $181(35,42)$ & $194(37,96)$ & $<0,001$ & 4,376 & $(2,090-9,163)$ \\
\hline 2 & $10(1,96)$ & $215(42,07)$ & $225(44,03)$ & & 6,757 & $(3,052-14,958)$ \\
\hline 3 & $22(4,31)$ & $70(13,70)$ & $92(18,00)$ & & 1,000 & Referência \\
\hline \multicolumn{7}{|c|}{ Pergunta 2: Qual sua área de atuação? } \\
\hline 1 & $3(0,59)$ & $169(33,07)$ & $172(33,66)$ & $<0,001$ & 1,000 & Referência \\
\hline 2 & $7(1,37)$ & $83(16,24)$ & $90(17,61)$ & & 0,211 & $(0,053-0,835)$ \\
\hline 3 & $13(2,54)$ & $144(28,18)$ & $157(30,72)$ & & 0,197 & $(0,055-0,704)$ \\
\hline 4 & $22(4,31)$ & $70(13,70)$ & $92(18,00)$ & & 0,057 & $(0,016-0,195)$ \\
\hline \multicolumn{7}{|c|}{ Pergunta 3: Você tem título de ortopedista e traumatologista pela Sociedade Brasileira de Ortopedia e Traumatologia (SBOT)? } \\
\hline 1 & $19(3,72)$ & $333(65,17)$ & $352(68,88)$ & $<0,001$ & 5,508 & $(2,831-10,719)$ \\
\hline 2 & $4(0,78)$ & $63(12,33)$ & $67(13,11)$ & & 4,950 & $(1,618-15,147)$ \\
\hline 3 & $22(4,31)$ & $70(13,70)$ & $92(18,00)$ & & 1,000 & Referência \\
\hline \multicolumn{7}{|c|}{ Pergunta 4: Você atualmente atua em unidade de urgência/emergência que atende trauma ortopédico do membro superior? } \\
\hline 1 & $37(7,24)$ & $399(78,08)$ & $436(85,32)$ & 0,5382 & 1,288 & $(0,575-2,886)$ \\
\hline 2 & $8(1,57)$ & $67(13,11)$ & $75(14,68)$ & & 1,000 & Referência \\
\hline
\end{tabular}

*Valor de $p$ referente ao teste qui-quadrado. Probabilidade modelada $1=$ Acerto.

Pergunta 1. 1: Completei minha residência há mais de 10 anos; 2: Completei minha residência há menos de 10 anos; 3: Sou residente de ortopedia e traumatologia.

Pergunta 2. 1: Cirurgia da mão; 2: Ortopedia geral; 3:Outras (Exemplos: cirurgia do joelho, quadril, coluna, etc.); 4:Sou médico residente de ortopedia e traumatologia.

Pergunta 3. 1: Sim; 2: Não; 3: Sou residente de ortopedia e traumatologia.

Pergunta 4. 1: Sim; 2: Não. 


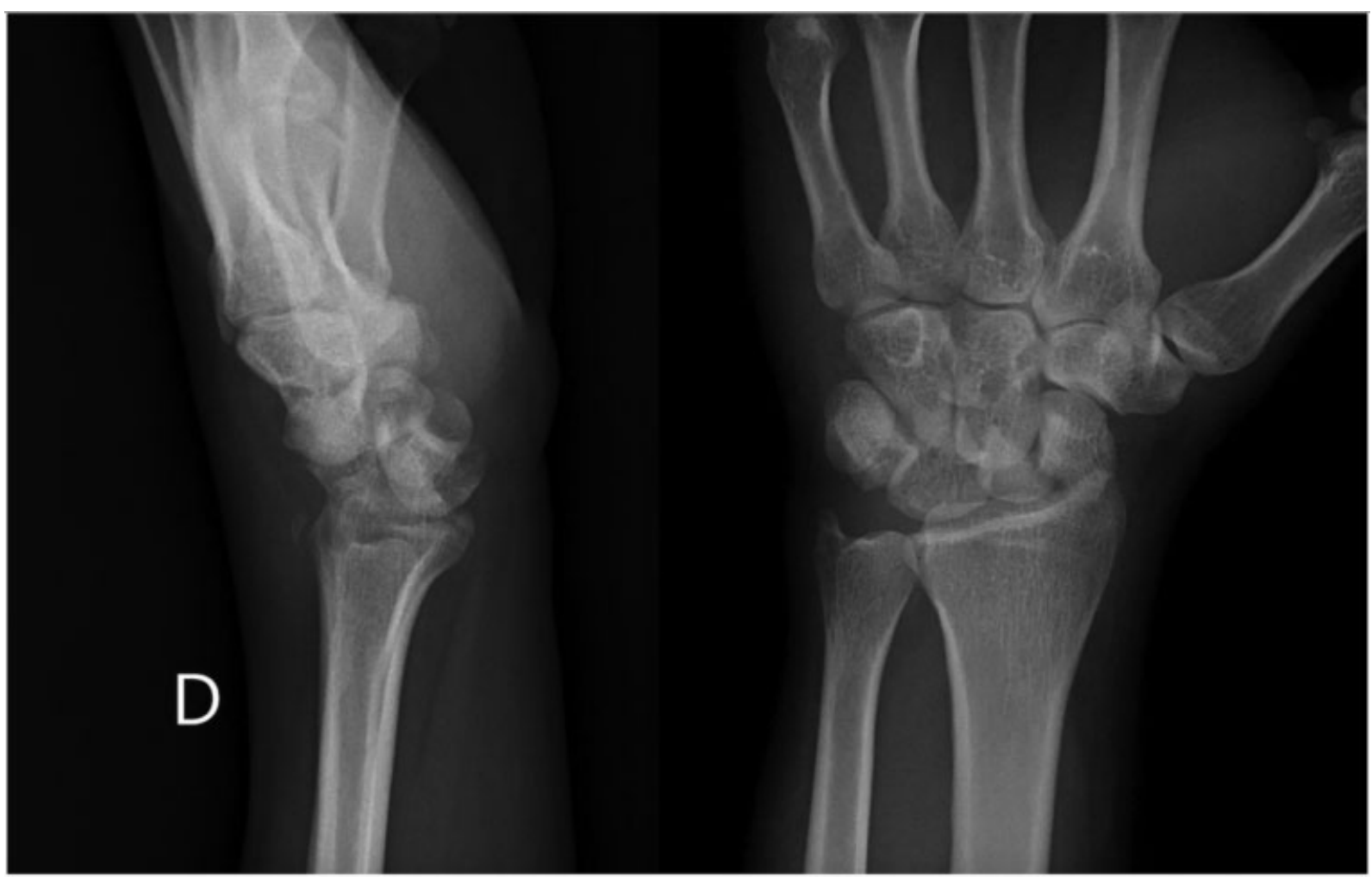

Fig. 3 Fratura-luxação transescafoperilunar estágio III de Mayfield.

Tabela 2 Tabela de frequências e regressão logística para a radiografia 2

\begin{tabular}{|c|c|c|c|c|c|c|}
\hline & \multicolumn{2}{|l|}{$\mathrm{RX2}$} & & & & \\
\hline & Erro (0) & Acerto (1) & & & & \\
\hline & $\mathrm{n}(\%)$ & $\mathrm{n}(\%)$ & Total & Valor de $p^{*}$ & Razão de chances bruta & Intervalo de confiança (95\%) \\
\hline \multicolumn{7}{|c|}{ Pergunta 1: Qual a sua graduação atual? } \\
\hline 1 & $0(0,00)$ & $194(37,96)$ & $194(37,96)$ & $<0,001$ & - & - \\
\hline 2 & $2(0,39)$ & $223(43,64)$ & $225(44,03)$ & & 9,182 & $(1,870-45,082)$ \\
\hline 3 & $7(1,37)$ & $85(16,63)$ & $92(18,00)$ & & 1,000 & Referência \\
\hline \multicolumn{7}{|c|}{ Pergunta 2: Qual sua área de atuação? } \\
\hline 1 & $0(0,00)$ & $172(33,66)$ & $172(33,66)$ & $<0,001$ & - & - \\
\hline 2 & $2(0,39)$ & $88(17,22)$ & $90(17,61)$ & & 3,624 & $(0,732-17,938)$ \\
\hline 3 & $0(0,00)$ & $157(30,72)$ & $157(30,72)$ & & - & - \\
\hline 4 & $7(1,37)$ & $85(16,63)$ & $92(18,00)$ & & 1,000 & Referência \\
\hline \multicolumn{7}{|c|}{ Pergunta 3: Você tem título de ortopedista e traumatologista pela Sociedade Brasileira de Ortopedia e Traumatologia (SBOT)? } \\
\hline 1 & $1(0,20)$ & $351(68,69)$ & $352(68,88)$ & $<0,001$ & 28,906 & $(3,509-238,094)$ \\
\hline 2 & $1(0,20)$ & $66(12,92)$ & $67(13,11)$ & & 5,435 & $(0,653-45,274)$ \\
\hline 3 & $7(1,37)$ & $85(16,63)$ & $92(18,00)$ & & 1,000 & Referência \\
\hline \multicolumn{7}{|c|}{ Pergunta 4: Você atualmente atua em unidade de urgência/emergência que atende trauma ortopédico do membro superior? } \\
\hline 1 & $7(1,37)$ & $429(83,95)$ & $436(85,32)$ & 0,5187 & 1,679 & $(0,342-8,241)$ \\
\hline 2 & $2(0,39)$ & $73(14,29)$ & $75(14,68)$ & & 1,000 & Referência \\
\hline
\end{tabular}

*valor de $p$ referente ao teste qui-quadrado. Probabilidade modelada $1=$ Acerto.

Pergunta 1. 1: Completei minha residência há mais de 10 anos; 2: Completei minha residência há menos de 10 anos; 3: Sou residente de ortopedia e traumatologia.

Pergunta 2. 1: Cirurgia da mão; 2: Ortopedia geral; 3:Outras (Exemplos: cirurgia do joelho, quadril, coluna, etc.); 4:Sou médico residente de ortopedia e traumatologia.

Pergunta 3. 1: Sim; 2: Não; 3: Sou residente de ortopedia e traumatologia.

Pergunta 4. 1: Sim; 2: Não. 
Europa em 1993. O índice encontrado foi de $25 \%$, sem discriminação entre as luxações simples e complexas. Em um estudo mais recente publicado na Turquia em 2018, foi encontrado 22.7\% de falha diagnóstica em 44 pacientes estudados com luxação ou fratura luxação perilunar do carpo. $O$ único fator de risco encontrado foi a inexperiência do ortopedista com a patologia. Dos cirurgiões que erraram o diagnóstico 70\% afirmaram ter sido a primeira vez que viram a patologia. ${ }^{13}$ Outro estudo recente, publicado em 2018, avaliou luxação e fraturaluxação perilunar em uma população de militares nos Estados Unidos encontrou uma falha no diagnóstico no primeiro atendimento em $27,5 \%$ dos casos. ${ }^{14}$ No presente estudo, o erro encontrado foi significativamente menor, 8,81\% para luxação perilunar simples e 1,76\% para fratura-luxação perilunar. Tais resultados podem ser devidos a um alto índice de suspeição dos ortopedistas, que pelo fato de estarem sendo observados e testados podem mudar seu comportamento pelo medo de errar. Um indicativo disso é que $38,49 \%$ das respostas para as radiografias normais apresentadas no questionário indicaram a presença de fratura ou luxação. Outro aspecto que interfere no índice de acerto diagnóstico é o horário de atendimento do paciente no serviço de emergência e a quantidade de descanso que o médico obteve. ${ }^{13,15}$ No estudo de Çolak et al. ${ }^{13}$ publicado na Turquia, $70 \%$ dos pacientes diagnosticados incorretamente deram entrada no serviço de emergência durante a madrugada. Pelo desenho do nosso estudo não foi possível analisar esta variável, que é uma particularidade da prática clínica.

Deve-se ainda considerar que o questionário respondido não corresponde à prática clínica, na qual é possível realizar uma anamnese e examinar o paciente, gerando um maior ou menor índice de suspeição para determinada patologia dependendo dos achados clínicos encontrados. Contudo, o estudo pode sugerir que o índice de falha diagnóstica não é tão elevado como se pensava, e que o estudo europeu de Herzberg et al. ${ }^{6}$ não é representativo da nossa realidade brasileira. Outra hipótese é que os erros podem estar decrescendo devido a uma melhoria na formação de médicos pelos serviços de residências de 1993, quando foi realizado o estudo de Herzberg, até a presente data. Um aspecto que corrobora essa última hipótese é que houve diferença significativa entre médicos residentes e ortopedistas já formados (23,9\% x 5,49\%), assim demonstrando que a residência de ortopedia e traumatologia está sendo eficaz em instruir os médicos residentes a identificar essa patologia nas radiografias simples.

Os cirurgiões de mão apresentaram os menores índices de erro, como já era esperado, devido à maior familiaridade com a afecção. Em contraste, a presença ou não do título de especialista reconhecido pela SBOT não representou diferenças no índice de acerto diagnóstico.

0 presente estudo indica a possibilidade do índice de erro diagnóstico para as luxações perilunares ser menor que o classicamente descrito na literatura. Entretanto, são necessários estudos com maior nível de evidência científica para poder realizar esta afirmação. Uma boa opção seria um estudo de caso controle multicêntrico, pois reflete com maior fidedignidade a prática clínica e permite um número satisfatório de casos, pois a luxação perilunar não é comum e um hospital isoladamente poderia enviesar o estudo pelas suas características demográficas específicas.

\section{Conclusão}

O índice de erro diagnóstico, utilizando radiografias simples, da luxação perilunar isolada foi de 8,91\% e da fratura-luxação foi $1,76 \%$, sugerindo que este método é eficaz e que o índice de erro pode não ser tão elevado quanto acreditava-se. Os cirurgiões de mão foram o grupo com menor índice de erro diagnóstico e os residentes de ortopedia demonstraram o maior índice de erro.

\section{Suporte Financeiro}

Não houve suporte financeiro de fontes públicas, comerciais, ou sem fins lucrativos.

Conflito de interesses

Os autores declaram não haver conflito de interesses.

\section{Referências}

1 Chiappini A, Alessandri M. Considerations on the bloodless reduction of a case of perilunar luxation of the carpus with fracture of the scaphoid and of the styloid apophysis of the radius. Ann Med Nav (Roma) 1964;69:399-410

2 Pappas ND III, Lee DH. Perilunate Injuries. Am J Orthop 2015;44 (09):E300-E302

3 Gilula LA. Carpal injuries: analytic approach and case exercises. AJR Am J Roentgenol 1979;133(03):503-517

4 Hildebrand KA, Ross DC, Patterson SD, Roth JH, MacDermid JC, King GJ. Dorsal perilunate dislocations and fracture-dislocations: questionnaire, clinical, and radiographic evaluation. J Hand Surg Am 2000;25(06):1069-1079

5 Mayfield JK, Johnson RP, Kilcoyne RK. Carpal dislocations: pathomechanics and progressive perilunar instability. J Hand Surg Am 1980;5(03):226-241

6 Herzberg G, Comtet JJ, Linscheid RL, Amadio PC, Cooney WP, Stalder J. Perilunate dislocations and fracture-dislocations: a multicenter study. J Hand Surg Am 1993;18(05):768-779

7 Herzberg G, Forissier D. Acute dorsal trans-scaphoid perilunate fracture-dislocations: medium-term results. J Hand Surg $[\mathrm{Br}]$ 2002;27(06):498-502

8 Meszaros T, Vögelin E, Mathys L, Leclère FM. Perilunate fracturedislocations: clinical and radiological results of 21 cases. Arch Orthop Trauma Surg 2018;138(02):287-297

9 Garcia-Elias M, Irisarri C, Henriquez A, et al. Perilunar dislocation of the carpus. A diagnosis still often missed. Ann Chir Main 1986;5 (04):281-287

10 Google. Google Forms. Disponível em: https://www.google.com/forms/about/. [Acesso em 1 de Julho de 2018]

11 Hosmer DW, Lemeshow S. Assessing the fit of the model. In: Applied Logistic Regression. New York: John Wiley and Sons; 1989:135-173

12 Scheffer M, Cassenote A, Guilloux AG, Biancarelli A, Bruno, Miotto A, Mainardi GM. Demografia Médica no Brasil 2018. São Paulo: Departamento de Medicina Preventiva da Faculdade de Medicina da USP; Conselho Regional de Medicina do Estado de São Paulo; Conselho Federal de Medicina; 2018

13 Çolak I, Bekler HI, Bulut G, Eceviz E, Gülabi D, Çeçen GS. Lack of experience is a significant factor in the missed diagnosis of perilunate fracture dislocation or isolated dislocation. Acta Orthop Traumatol Turc 2018;52(01):32-36

14 Dunn JC, Koehler LR, Kusnezov NA, et al. Perilunate Dislocations and Perilunate Fracture Dislocations in the U.S. Military. J Wrist Surg 2018;7(01):57-65

15 Orton DI, Gruzelier JH. Adverse changes in mood and cognitive performance of house officers after night duty. BMJ 1989;298 (6665):21-23 